\title{
Investigation of Sensors Based on ITO Nanofilms in SPR-Devices
}

\author{
Volodymyr Maslov*, Glib Dorozinsky, Nataliya Kachur \\ Department of Physics and Technological Bases of Sensory Materials, V. Lashkaryov Institute of Semiconductor Physics NAS of Ukraine, \\ Kyiv, Ukraine
}

Email address:

vpmaslov@ukr.net (V. Maslov), gvdorozinsky@ukr.net (G. Dorozinsky), natalykachur@gmail.com (N. Kachur)

${ }^{*}$ Corresponding author

\section{To cite this article:}

Volodymyr Maslov, Glib Dorozinsky, Nataliya Kachur. Investigation of Sensors Based on ITO Nanofilms in SPR-Devices. American Journal of Optics and Photonics. Vol. 4, No. 3, 2016, pp. 20-24. doi: 10.11648/j.ajop.20160403.11

Received: September 23, 2016; Accepted: September 30, 2016; Published: October 19, 2016

\begin{abstract}
Devices based on surface plasmon resonance (SPR) are used to determine the refraction index (RI) of liquids and gases. In these cases, as a sensitive element (SE), they use precious materials. It is also known that RI of an investigated substance (analyte) depends on temperature, which brings in some errors into results of measurements. The existing construction solutions for controlling and adjusting the analyte temperature possess a number of deficiencies, namely: high inertness and consumption power. The aim of this work was to study the possibility of using ITO films $\left(\operatorname{In}_{2} \mathrm{O}_{3}\right.$-SnO) as SE and thin-film heaters in SPR devices to enhance their accuracy. For the first time, excitation of SPR by using the wavelength $650 \mathrm{~nm}$ in ITO films has been proved experimentally. It has been also shown that using the heaters based on ITO films reduces the error of measuring the analyte RI by 1.8 times and the consumed power - by 2.3 times as compared with the existing heaters of the resistive type that are used now in SPR devices.
\end{abstract}

Keywords: Thin Film Coating, Plasmonics, Biosensor, Sensor

\section{Introduction}

The leading tendencies in development of analytic devices require implementation of new physical methods for measurements that are based on up-to-date achievements in science and technique. The main requirements to these methods are as follows: increasing their accuracy and sensitivity, shortening the time for measurements, reducing the volume of studied substances. It is known that optical methods possess a high operation speed and enable to reach high accuracy and sensitivity in measurements. One of the promising optical methods for analysis of various compounds and micro-objects as well as processes at the molecular level is the refractometric method based on SPR phenomenon. The respective devices (SPR-devices) are mainly designed using chemical and biological sensors that consist of SE and some physical transducer. As compared with traditional measuring methods, the SPR method provides possibility to study processes of molecular interaction in micrometer-thickness layers in the real-time scale; low value of the sample volume required for measurements (less than $10 \mu \mathrm{l}$ ); the method does not require any markers or fluorescent labels for studying the analyte [1].

The SPR-devices are widely used in scientific researches, in medicine, pharmacology and ecological monitoring [2-6]. Their SE use precious materials, namely: silver and gold [7]. Metal oxides are used here as additional layers covering the gold or silver layers of SE for enhancement of adsorption properties and waveguide amplification of response to changes in the analyte RI [8, 9]. Therefore, applying the advanced materials for SE and enhancement of accuracy inherent to these devices seems to be a topical task.

One of the promising materials for nanoplasmonics is ITO $\left(\mathrm{In}_{2} \mathrm{O}_{3}-\mathrm{SnO}\right)$ [10]. Composite thin ITO films are mainly used as transparent electrodes for solar cells [11, 12]. Availability of conduction in ITO makes it possible to excite SPR in them.

As shown in the previous works [13, 14], account of the 
temperature factor is essential for enhancement of accuracy in measurements when using the SPR-device.

To decrease the influence of temperature factor on results of measuring RI, they apply the methods of thermal stabilization and thermal compensation. Thermal stabilization means keeping the set temperature of a device as well as reservoirs with analytes [15], which is provided by resistive heaters and/or Peltier elements, To widen the range of temperature control and fast response, one should increase the electric power consumed by them.

However, it results in increasing dimensions and power consumed by SPR-devices as well as time for transfer heat from a heater. Thermal compensation decreases only the temperature drift, and it is efficient only in stationary temperature regimes [16].

Investigated in this work were sensors based on ITO nanofilms in SPR-devices.

\section{Devices and Methods of Investigation}

Investigation of the temperature influence on accuracy of measurement results and power consumption of ITO thin film heaters was performed using the SPR-device «Plasmon-6» [17]. The devices of "Plasmon" series are computer-controlled optoelectronic small-scale two-channel refractometers based on SPR phenomenon for determination of the SPR curve or total internal reflection (TIR) envelope shift inherent to the studied analyte. Operation surface of the «Plasmon» sensitive element is formed from a gold film with the thickness 48 to 52 $\mathrm{nm}$ on flint glass substrate. As a source for excitation, there used is p-polarized light from the semiconductor GaAs injection laser diode with the wavelength $650 \mathrm{~nm}$.

Data for plotting the SPR curve (TIR envelope) are obtained by rotating the prism and simultaneous measuring the intensity of light reflected from the sensitive element. The channels are formed by the measuring cell 12 made of polymethylmetacrylate, which is mounted on the sensitive element via the silicone spacer. To excite surface plasmons in both channels, light from the laser diode is separated by two beams with the prism 5. Control and stabilization of the light intensity are provided with the photodiode 6 and device electronics, respectively. Calibration of the absolute angle is performed with the photodiode 4 and diaphragm 2 (slot width is close to $100 \mu \mathrm{m}$ ) by measuring the intensity of light reflected from the prism front face. Measurements of the laser light intensity and calibration of the angle are provided with the separating plate 3 and diaphragm 10 .

Except prism, all the elements of this optical scheme, sensitive element and measuring cell form a separate assembly - the optical unit. Angular scanning and determining the minimum position of the SPR curve are provided within the range of angles 38 to 71 degrees for rotation of the prism on the platform-holder with the step-motor and reducer.

Composite thin films ITO $\left(\mathrm{In}_{2} \mathrm{O}_{3}-20 \%\right.$; SnO-80\%) were prepared using magnetron sputtering onto glass substrates of the thickness $1 \mathrm{~mm}$ and dimensions $18 \times 14 \mathrm{~mm}$. The thickness of deposited layer was determined using atomic force microscopy. At the final stage of formation, this thickness reached the value $50 \mathrm{~nm}$.

Reflection characteristics for these films were measured at the angles larger that the angle of total internal reflection (TIR). It allowed checking the possibility to excite SPR in these films at the wavelength lower than $1 \mu \mathrm{m}$, namely $650 \mathrm{~nm}$.

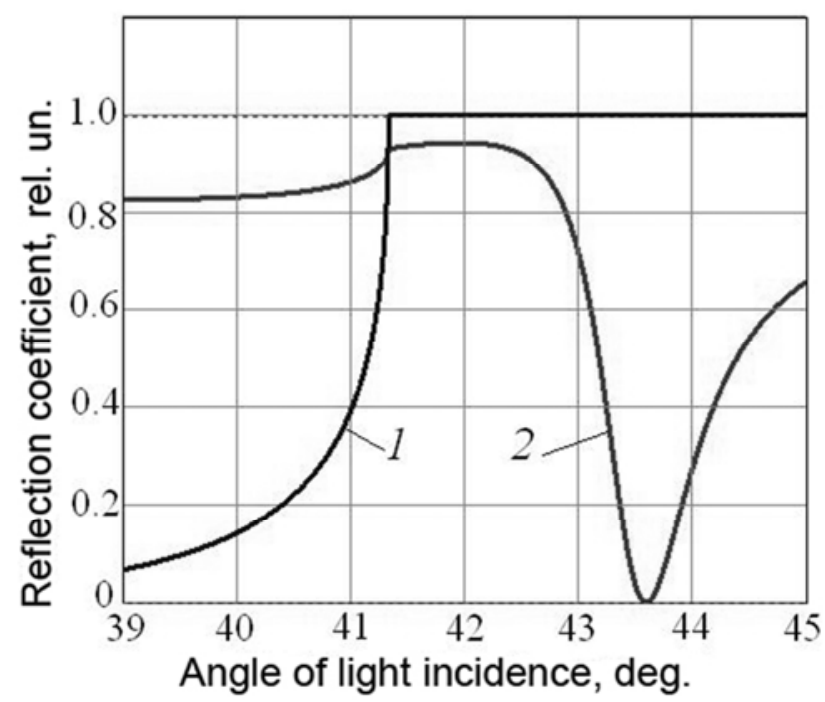

(a)

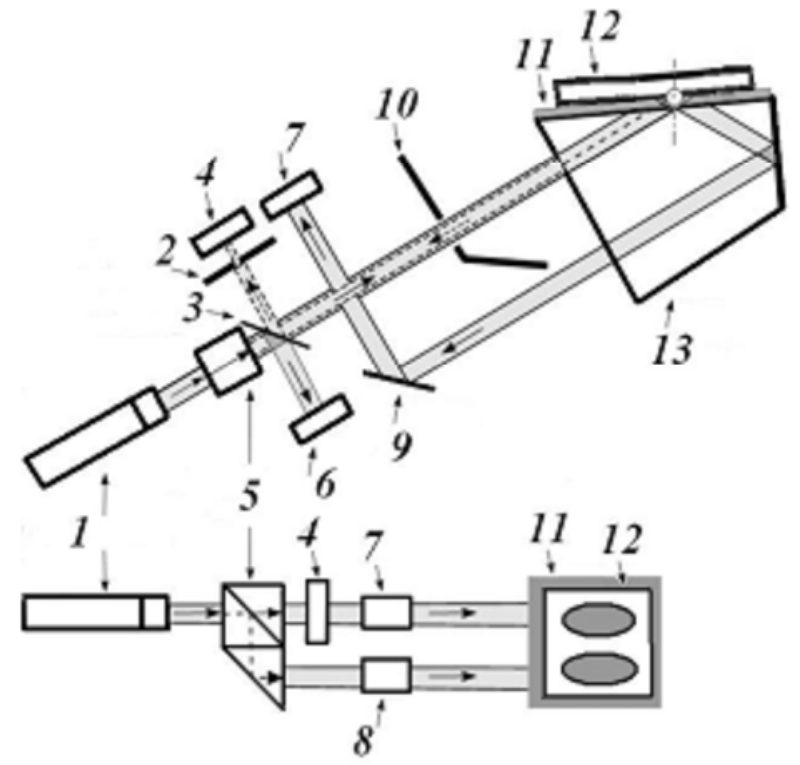

(b)

Figure 1. (a) SPR curve (1), TIR envelope (2) and (b) optical scheme of the device «Plasmon-6». Designations of elements see in the text.

When measuring reflection under SPR conditions, the glass substrate with thin ITO film should possess high transparency for the operation wavelength. Therefore, transmission spectra of the respective substrates were measured within the wavelength range $300 \ldots 1100 \mathrm{~nm}$ by using the spectrophotometer UNICO 4802 UV/VIS.

When studying the thin ITO films as heaters, we 
determined the consumed power by using the measured current-voltage characteristics with simultaneous measuring the temperature augmentation caused by their self-heating. The voltage on the sample was applied from a power supply $(10 \mathrm{~W})$ with fine adjustment of the output voltage within the range 1.5 to $31 \mathrm{~V}$ and uncertainty $\pm 0.1 \mathrm{~V}$. Electrical contact between the power supply and ITO film was provided using copper plates of the thickness $0.3 \mathrm{~mm}$ and dimensions $18 \times 3 \mathrm{~mm}$. These plates were connected with the probes of power supply.

To measure the voltage and current through the sample, we used the digital voltmeter DT-830B with the error of measurements $0.5 \%$. The temperature was determined using the voltmeter DT- 838 with the thermocouple being in contact with the composite film surface. The error of temperature measurements was no higher than $\pm 0.2 \mathrm{~K}$.

Changes in the air refraction index were determined using the device Plasmon- 6 and observing the shift of the operation point located at the slope of the reflection characteristic before the TIR angle at the boundary film-air. The device allowed measuring the changes in the intensity of light reflected from the boundary in the mode Slope, the substrate being heated by electric current. Further, the measured intensity values were re-calculated into refraction index values by using special software.

Used here are temperature-sensitive elements M222Pt100 (Heraeus Sensor Technology, Germany) with dimensions $2 \times 2 \times 1.2 \mathrm{~mm}$, linear temperature characteristic within the range from -203 up to $+773 \mathrm{~K}$ and initial resistance $1 \mathrm{kOhm}$ at the temperature $293 \mathrm{~K}$ (DIN EN6075 accordingly to IEC 751).

The same measurements were performed for the glass substrate without ITO film but heating the studied substance by using a constantan wire resistive heater (resistance 42 $\mathrm{Ohm}$ at the temperature $\mathrm{T}=20^{\circ} \mathrm{C}$ ) built-in into the measuring cell of the SPR-device, the supply voltage of the heater being $12 \mathrm{~V}$.

Comparison of the consumed power and the error of measurements caused by the temperature drift of the operation point were performed at the given temperature $36.6 \pm 0.2^{\circ} \mathrm{C}$.

The error of measurements was determined as the value of operation point drift, which was expressed using the values of refraction index for the studied air for the interval of measurements equal to $1 \mathrm{~min}$.

\section{Results of Experiments and Discussion}

The results of our experiments have shown that SPR is excited in ITO films at the wavelength of exciting radiation $650 \mathrm{~nm}$, which is confirmed by the characteristic minimum in the reflection curve for the angles higher than the TIR angle at the boundary film - air (55.175 degree, Fig. 2).

The studied composite films possess high transparency (Fig. 3) with the peak in the transmission spectrum in the middle of the visible range $(575 \mathrm{~nm})$.

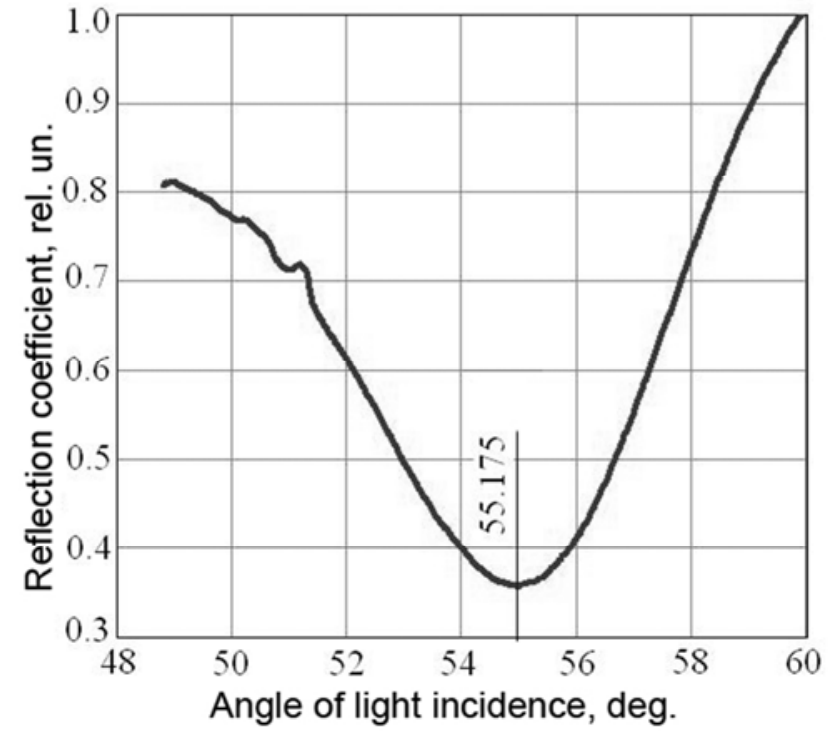

Figure 2. Dependence of the reflection coefficient on the angle of light incidence at the boundary ITO film-air under excitation of SPR in this film.

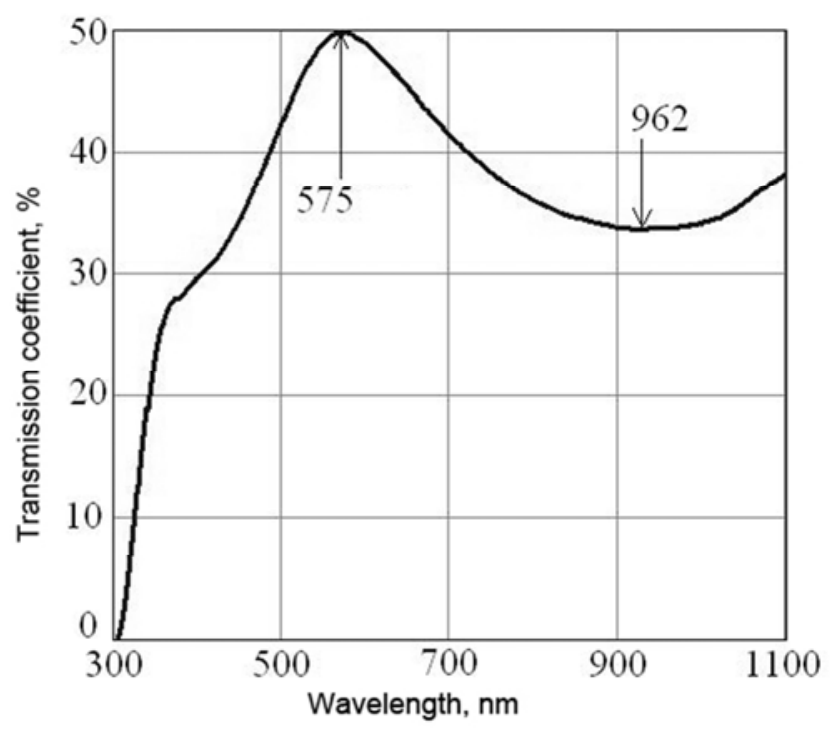

Figure 3. Transmission spectrum of the ITO film with the thickness $50 \mathrm{~nm}$ on a glass substrate.

Using the current-voltage characteristics of ITO films, we calculated the dependence of the power that is released in the film sample on the voltage applied to it (Fig. 4). When the voltage reached its maximum value $30 \mathrm{~V}$ and consumed power $-1.2 \mathrm{~W}$, the film and substrate were heated from $21.5^{\circ} \mathrm{C}$ up to $60.5^{\circ} \mathrm{C}$ for only $5 \mathrm{~min}$ (Fig. 5).

When measuring the refraction index for air, the substrate with the ITO layer was mounted on the prism operation face by using immersion liquid ( $100 \%$ glycerin). In this case, the film and substrate were heated to the lower temperature $\left(54.8^{\circ} \mathrm{C}\right)$ for $20 \mathrm{~min}$, the consumed power being $1.5 \mathrm{~W}$ and voltage $-30 \mathrm{~V}$, which is explained by additional heat dissipation in prism material. At the same time, to heat the only glass substrate (without ITO film and placed on the prism face) with the resistive heater up to $55^{\circ} \mathrm{C}$, it requires the power $3.4 \mathrm{~W}$ and voltage $12 \mathrm{~V}$. 
Thus, using the film heater enabled us to reduce power consumption by more than 2 times.

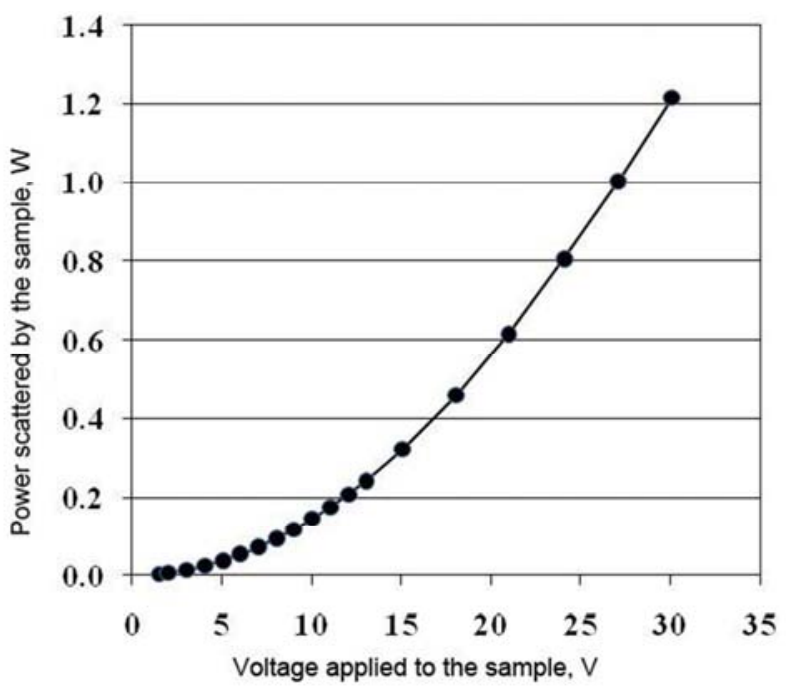

Figure 4. Dependence of the power released from the ITO film on the voltage applied to it.

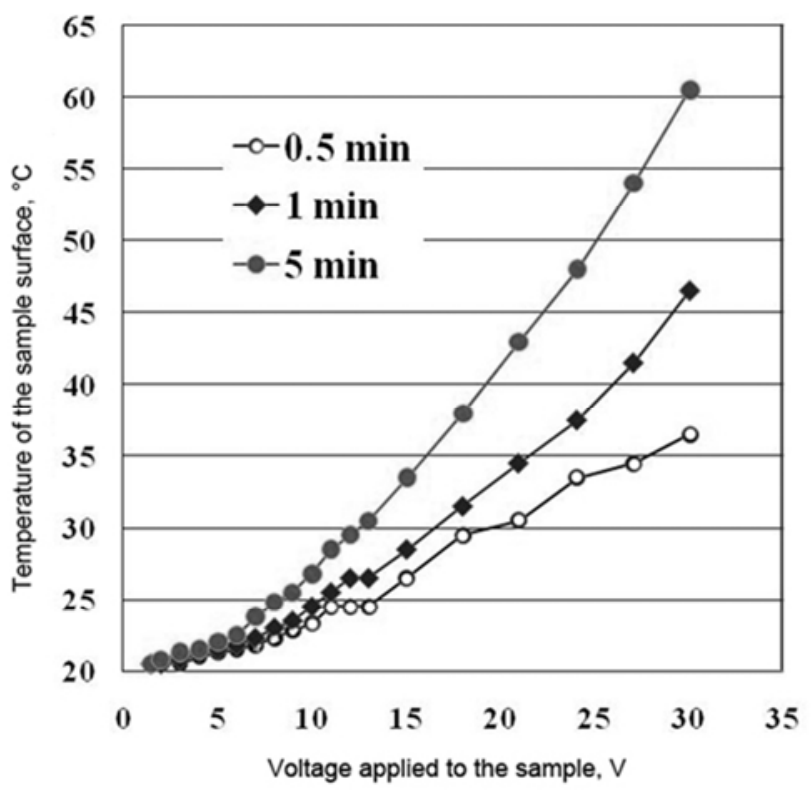

Figure 5. Dependence of the surface temperature for the ITO film on the voltage applied to it.

Our measurements of the temperature drift observed for the operation point have shown that, using the film heater, this drift is by 1.8 times lower than that for the resistive heater, i.e., $3.6 \cdot 10^{-6} \mathrm{~min}^{-1}$ against $6.5 \cdot 10^{-6} \mathrm{~min}^{-1}$. This fact is related with lower inertness of the processes of heat transfer from thin film as compared with that of the bulk heater.

\section{Conclusions}

A. For the first time, shown in this paper is the possibility to excite SPR in thin ITO films by using the wavelength $650 \mathrm{~nm}$ and applying these films as sensitive elements for SPR-devices.
B. It has been proved experimentally that applying the thin-film heating elements based on ITO in SPR-devices allows to reduce the temperature drift of operation point and, consequently, the error in measurements of the refraction index of the studied substance by 1,8 times as compared with that of bulk resistive heaters.

C. The thin-film heaters based on ITO consume more than 2 -fold lower power in the case of the same heating the studied substance as compared with that for bulk resistive heaters.

\section{References}

[1] J. Homola, Surface Plasmon Resonance Based Sensors, Springer Series on Chemical Sensors and Biosensors, Series Editor: O. S. Wolfbeis, Springer-Verlag, Berlin Heidelberg, 2006, pp. 45-67.

[2] I. D. Voitovych, S. G. Korsunskyi, Sensors based on plasmon resonance: principles, technologies, applications, Stal, Kyiv, 2011, 534 p.

[3] N. Ya., Gridina, V. P. Maslov, Yu. V. Ushenin, Tumor-associated inflammation and cerebral gliomas, Lambert Academic Publishing, Saarbrucken, 2013, 196 p.

[4] G. V. Dorozinsky, V. P. Maslov, Yu. V. Ushenin, Sensory devices based on surface plasmon resonance, NTUU "KPI", Kyiv, 2016, 264 p.

[5] J. Svitel, A. Dzgoev, K. Ramanathan, B. Danielsson, "Surface plasmon resonance based pesticide assay on a renewable biosensing surface using the reversible concanavalin A monosaccharide interaction", Biosensors and Bioelectronics, SCIRP, pp. 411-415, 2000.

[6] Dorozinsky, G. V., M. V. Lobanov, V. P. Maslov, "Detection of methanol vapors by using the method of surface plasmon resonance", East-Europian Journal of Advanced Technologies, pp. 4-7, 2015.

[7] Yu. M. Shirshov, A. V. Samoylov, S. A. Zinyo, E. R. Surovceva and V. Mirskiy, "Bimetallic layers increase sensitivity of affinity sensors based on surface plasmon resonance", Sensors, pp. 62-70, 2002.

[8] Yu. V. Ushenin, R. V. Khristosenko, A. V. Samoylov, Yu. S. Gromovoi, E. B. Kaganovich, E. G. Manoilov, S. O. Kravchenko, B. A. Snopok, "Thin films of porous aluminum oxide obtained using pulsed laser deposition for surface plasmon-polariton sensor structures", Fizika i khimiya tverdogo tila, pp. 259-264, 2012.

[9] Nan-Fu Chiu, Yi-Chen Tu, Teng-Yi Huang, "Enhanced Sensitivity of Anti-Symmetrically Structured Surface Plasmon Resonance Sensors with Zinc Oxide Intermediate Layers", Sensors, pp.170-187, 2014.

[10] A. U. Akrajas, I. Iwantono, A. Ariyanto, M. Mat Salleh, M. Oyama, "Gold nanonetwork film on the ITO surface exhibiting one-dimensional optical properties", Nanoscale Research Letters, Springer, pp. 252- 260, 2012.

[11] Lung-Chien Chen, Jhih-Chyi Chen, Cheng-Chiang Chen and Chun-Guey Wu, "Fabrication and Properties of High-Efficiency Perovskite/PCBM Organic Solar Cells", Nanoscale Research Letters, Springer, pp. 312- 317, 2015. 
[12] D. A. Grynko, A. N. Fedoryak, P. S. Smertenko, O. P. Dimitriev, N. A. Ogurtsov, A. A. Pud, "Hybrid solar cell on a carbon fiber", Nanoscale Research Letters, Springer, pp. 265273,2016 .

[13] S. K. Ozdemir and G. Turhan-Sayan, "Temperature Effects on Surface Plasmon Resonance: Design Considerations for an Optical Temperature Sensor", Journal of light wave technology, pp. 805-815, 2003.

[14] K. Lin, "Numerical and experimental investigations of temperature effects on the surface plasmon resonance sensor", Chinese Optics Letters, pp. 428-431, 2009,.

[15] G. Dorozinsky, V. Maslov, A. Samoylov, Yu. Ushenin,
"Reducing measurement uncertainty of instruments based on the phenomenon of surface plasmon resonance", American Journal of Optics and Photonics, pp. 17-22, 2013.

[16] Yu. V. Ushenin, V. P. Maslov, T. A. Turu, N. V. Kachur, "Application of temperature sensors for improving the device based on the phenomenon of surface plasmon", Sensor Electronics and Microsystem Technologies, Odessa, pp. 33-40, 2016.

[17] Yu. V. Ushenin, A. V. Samoylov, R. V. Khristosenko. Enhancement of sensitivity inherent to sensors based on surface plasmon resonance for measuring the medium refraction index. Texnologiya i konstruirovaniye elektronnoj apparatury, Politechperiodika, Odessa, pp. 12-14, 2011. 